\title{
An all-optical modulator based on a stereo graphene-microfiber structure
}

\author{
Jin-Hui Chen, Bi-Cai Zheng, Guang-Hao Shao, Shi-Jun Ge, Fei Xu and Yan-Qing Lu
}

An in-line, all-optical fiber modulator based on a stereo graphene-microfiber structure (GMF) utilizing the lab-on-rod technique was demonstrated in this study. Owing to its unique spring-like geometry, an ultra-long GMF interaction can be achieved, and a modulation depth of $\sim 7.5 \mathrm{~dB}(\sim 2.5 \mathrm{~dB})$ and a modulation efficiency of $\sim 0.2 \mathrm{~dB} \mathrm{~mW}^{-1}\left(\sim 0.07 \mathrm{~dB} \mathrm{~mW}^{-1}\right)$ were demonstrated for two polarization states. The modulation depth and modulation efficiency are more than one order of magnitude larger than those of other graphenemicrofiber hybrid all-optical modulators, although at the cost of a higher insertion loss. By further optimizing the transferring and cleaning process, the upper limit of the modulation depth is mainly determined by the loss from the intrinsic absorption, which depends on the light-graphene interaction. Then, the modulator can quickly switch between the on-state and the off-state with a theoretically maximized modulation depth of tens of decibels. This modulator is compatible with the current fiber-optic communication systems and may be applied in the near future to meet the impending need for ultrafast optical signal processing.

Light: Science \& Applications (2015) 4, e360; doi:10.1038/lsa.2015.133; published online 4 December 2015

Keywords: graphene; microfiber; optical modulation; polarization

\section{INTRODUCTION}

Two-dimensional materials, especially graphene, have attracted global interest ${ }^{1}$, and many extraordinary electrical, mechanical, and optical properties have been reported. Regarding their exceptional optical properties, for example, $2.3 \%$ linear absorption (related to the fine structure constant) ${ }^{2}$, saturable absorption, and a tunable Fermi level (either by electrical gating ${ }^{3,4}$, chemical doping ${ }^{5,6}$, or even plasmon-induced doping $^{7}$ ) have been demonstrated for a number of broadband optical devices based on graphene in both normal-incidence and waveguideintegrated configurations, such as mode-locked lasers ${ }^{8}$, ultrafast photodetectors $^{9-11}$, and modulators ${ }^{12,13}$. Among these, the optical modulator is a key device for optical communication that converts optical or electrical data into optical signals. A high modulation speed, sufficient modulation depth, and large optical bandwidth are indispensable for an optical modulator. Although tuning the Fermi energy by using electric gating on graphene to achieve modulators of gigahertz-level modulation speed has been demonstrated ${ }^{12,13}$, it is difficult to further improve on the modulation speed due to limitations of the response time of the bias circuit. All-optical modulation can overcome the limitations on the modulation rate by using one light beam to control the transmission of another light beam. This type of modulation may avoid electricaloptical-electrical conversions, thereby rendering the modulation process faster and less noisy. Several papers have reported all-optical modulation schemes with a high speed of $200 \mathrm{GHz}$ based on graphene-microfiber-integrated devices by covering or wrapping a graphene sheet on a straight microfiber. The modulation depth or modulation efficiency is relatively low because a relatively low insertion loss is desirable in these schemes ${ }^{14,15}$. Higher modulation depth or modulation efficiency can be achieved with a higher loss by enhancing the lightgraphene interaction because of the trade-off among the modulation depth (modulation efficiency), the insertion loss and even the light traveling time. Moreover, it is challenging to handle such a thin microfiber directly for graphene integration with a sufficient length and strength of interaction for practical applications.

Recently, based on the rod-wrapping technique ${ }^{16}$, we developed a reliable approach to fabricating graphene-microfiber structure (GMF)-integrated devices by wrapping a microfiber on a graphenemodified rod. This method is much simpler than the conventional integration techniques because we only need to coat a small piece of graphene on a thick rod, rather than a thin microfiber. Theoretically, with a strong evanescent field, the GMF interaction length can be arbitrarily increased using a spring shape of as many turns as is spatially possible. Owing to the extremely asymmetrical cross section of such a stereo GMF device and the nature of the graphene-light interaction $^{12}$, different types of losses occur in the two fundamental modes in this hybrid structure. A broadband polarizer can be naturally integrated into such a device ${ }^{17}$. The electric fields of the fundamental modes are parallel and vertical to the surface of the supporting rod. In the present study, we show that the stereo GMF structure can also operate as a polarization-dependent, all-optical graphene modulator at approximately $1550 \mathrm{~nm}$, with a very high modulation depth $(\sim 7.5 \mathrm{~dB})$ and high modulation efficiency $\left(\sim 0.2 \mathrm{~dB} \mathrm{~mW}^{-1}\right)$, which are more than one order of magnitude larger than those reported previously $^{15}$. In fact, the modulation depth can be increased to tens of decibels if we can fabricate pure and clean GMF devices by improving the transferring and cleaning process. This type of fiber-integrated, 
in-line, all-optical modulator has great potential in fiber optical communication, in which there are demands for high-speed, wideband, low-cost, and integrated methods to modulate information.

\section{MATERIALS AND METHODS}

Figure 1 shows the fabrication process of the stereo GMF device. To prevent the loss induced by the relatively high index of the rod (polymethyl methacrylate or PMMA of diameter $\sim 2 \mathrm{~mm}$ ), a thin low-index Teflon layer (Teflon AF 601S1-100-6, DuPont, tens of micrometers in thickness with a refractive index of $\sim 1.31$ ) was dip-coated on the rod's surface and dried in air for several hours. The graphene on the Cu foil (Six Carbon Technology) was first spin-coated with a 4\% PMMAanisole solution and then dried in an oven for 30 minutes at $60{ }^{\circ} \mathrm{C}$. After dissolving the $\mathrm{Cu}$ foil using aqueous $\mathrm{FeCl}_{3}$ at $1 \mathrm{~mol} \mathrm{~L}{ }^{-1}$ and cleaning the sample in deionized water (DI), the PMMA/graphene film was removed from the DI water using a rod. The surface tension led to the film tightly encapsulating the rod when the film left the DI water. Then, the rod with the graphene/PMMA film was heated in an oven for 30 minutes at $80{ }^{\circ} \mathrm{C}$, and the PMMA film was removed by acetone. Finally, a tapered microfiber was wrapped onto the graphene-functionalized rod.

Raman spectroscopy was carried out by using the $488 \mathrm{~nm}(2.54 \mathrm{eV})$ lines of an Ar ion laser as excitation sources. The laser beam was focused onto the sample using a $50 \times$ microscope objective lens $(0.8$ N.A.), and the scattered light was analyzed by a monochromator. The Raman spectrum clearly shows the single-layer nature of the transferred graphene ${ }^{18}$, as shown in Figure 2. In our experiment, we chose a microfiber with a diameter of $\sim 3 \mu \mathrm{m}$, and there were two microfiber coils on a rod, which implies that the light-graphene interaction length was $\sim 12 \mathrm{~mm}$. The microfiber in our experiment was tapered from a conventional single mode fiber by utilizing a flame-brushing technique.

The all-optical modulation mechanism is based on the Pauli blocking effect, as illustrated in Figure 3a. When a high-power pump light at a short wavelength excites dense carriers in graphene from the valence band to the conduction band, the non-equilibrium carrier distribution broadens and equilibrates with the intrinsic carrier population through carrier-carrier scattering. The scattering process lasts for only hundreds of femtoseconds. The subsequent cooling and decay of the hot distribution through carrier-phonon scattering occur on a time scale of picoseconds ${ }^{19}$. Carriers relaxing from the conduction band leads to a band-filling effect, which results in a significant reduction in the graphene absorption for light of a greater wavelength. In this manner, we can use a high-power pump light source to switch a weak probe light source. As we can see above, the photon-excited carrier's relaxation process is dominated by carrier-phonon scattering, which lasts for several picoseconds. In other words, the all-optical modulation speed can theoretically reach hundreds of gigahertz. This has been experimentally demonstrated by using ultrafast optical pumpprobe spectroscopy to measure the decay time of graphene carriers coated on a straight microfiber ${ }^{14}$. Moreover, the modulation depth increases with the light-graphene interaction length and strength, which are greatly enhanced in the stereo GMF platform. However, inhomogeneous interaction in the large-length, light-graphene interaction inevitably leads to an inhomogeneous graphene response and thus limits its fast response. As a result, a trade-off should exist between the modulation depth and response time, as discussed below.
1. PMMA on graphene grown on copper foil

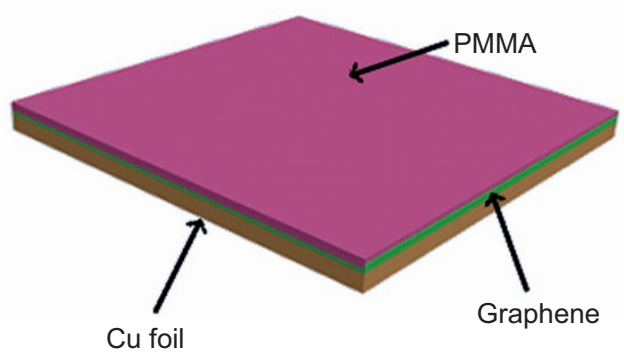

4. GMF schematic structure

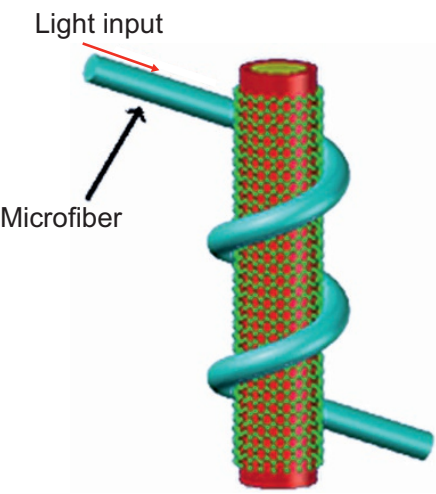

2. Graphene on PMMA film wrapped onto supporting rod

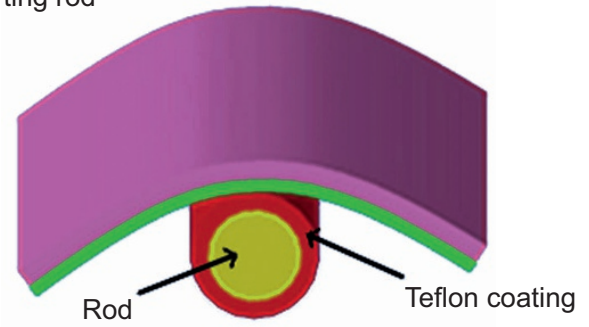

Remove the PMMA/graphene film from DI water using a rod. Dry the sample in an oven and dissolve the PMMA film in acetone.
3. Graphene on rod

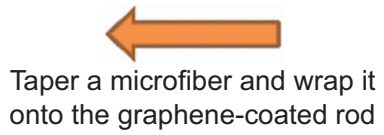

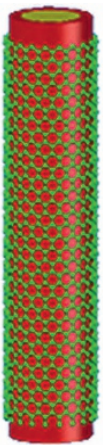

Figure 1 Fabrication process of the stereo hybrid GMF device. Panels 1-3 demonstrate the detailed steps for transferring graphene onto a rod. Panel 4 shows a schematic structure of the GMF device. 


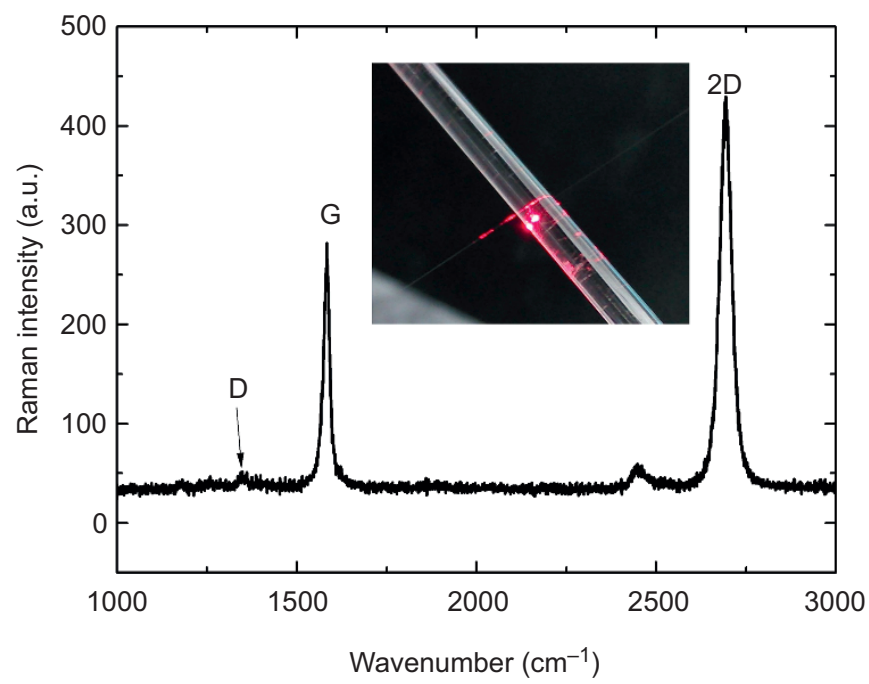

Figure 2 Raman spectrum of a monolayer graphene film. Inset, camera image of the as-fabricated GMF device, launched with a $650 \mathrm{~nm}$ light source. The strong scattering region indicates the graphene-microfiber interaction region.

\section{RESULTS AND DISCUSSION}

In our experiment, we first used a continuous-wave (CW) pump light source at $980 \mathrm{~nm}$ (OPEAK, Pump-LSB-980-500-SM) to measure the static modulation characteristics of our polarization-dependent stereo GMF platform, as shown in Figure 3b. Here, we call it "static" to distinguish it from the later pulse pump probe experiment, which is called "dynamic". Because the amplified spontaneous emission (ASE) light source (Connet C-ASE Optical Source) is non-polarized, a linear polarizer and a half-wave plate are incorporated between the light source and fiber wavelength-division multiplexer (WDM) to selectively excite different eigen-modes of our GMF with the same launching power. The ASE power input in our experiment was fixed at $\sim 300 \mu \mathrm{W}$. As shown in Figure 3c, when the pump light is switched off, the output intensity difference between the low absorptionloss mode (LAM) and the high absorption-loss mode (HAM) is approximately $15 \mathrm{~dB}$ at $1550 \mathrm{~nm}$, which is attributable to the broken symmetry of the GMF geometry and the absorption nature of graphene. The difference can be reduced or even eliminated by increasing the pump power. The small ripple superposed in the output spectra is attributable to the interface interference caused by the polarizer.

As the pump power increases, the output intensities of the HAM and LAM also increase because of the saturable absorption properties of graphene, as shown in Figure 3c. Here, we define the modulation depth as the transmission change of one polarization mode in the a

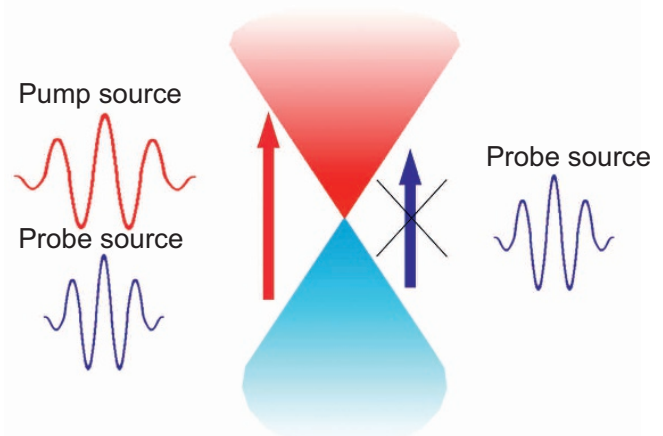

b
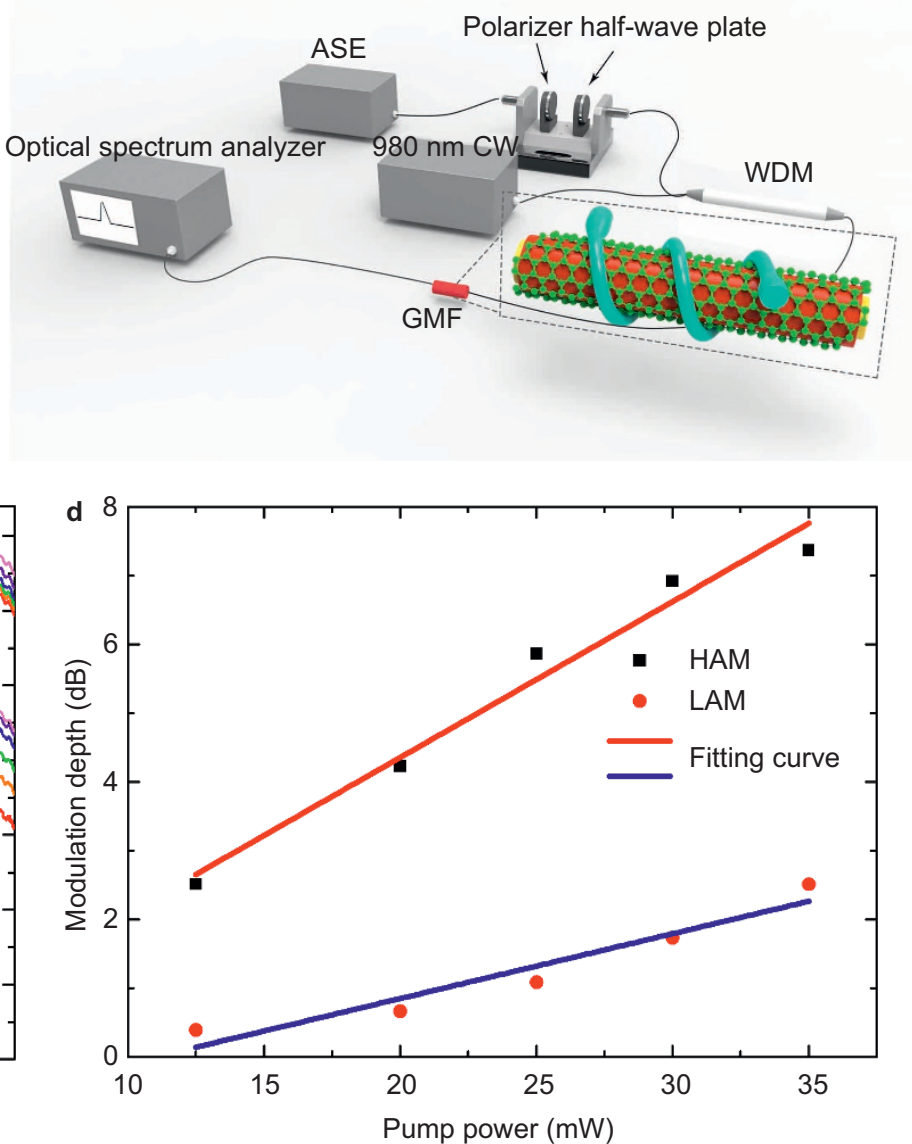

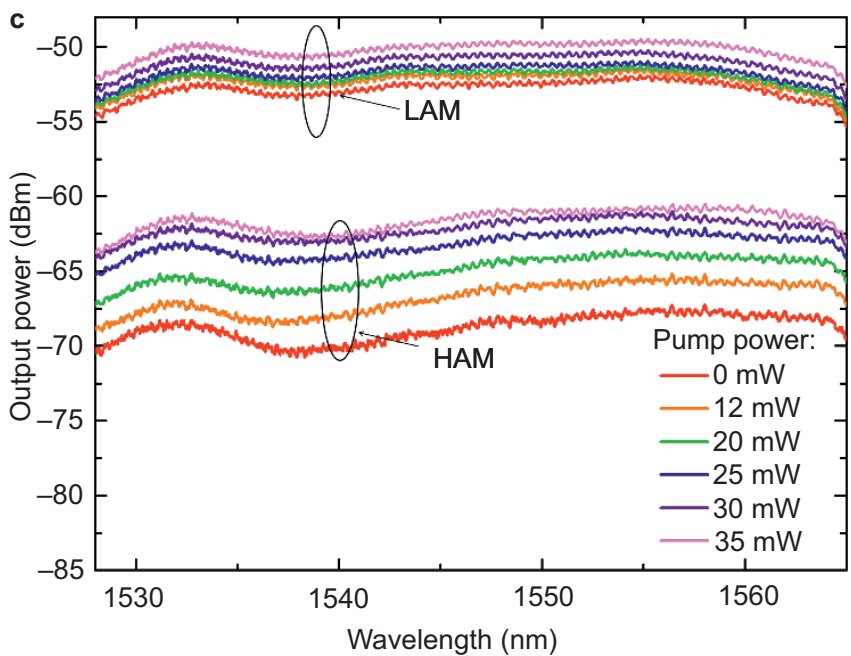

Figure $3 \mathrm{CW}$-pump-based modulation measurement system and results. (a) Schematic illustration of the all-optical modulation mechanism. (b) CW pump probe measurement system. (c) Output spectra of the stereo GMF device with a 980-nm pump source with different powers for the LAM and HAM. (d) Changes in modulation depth with different pump powers for 1550-nm probe wavelength, extracted from (c). The blue and red lines are numerical fitting curves. 
a

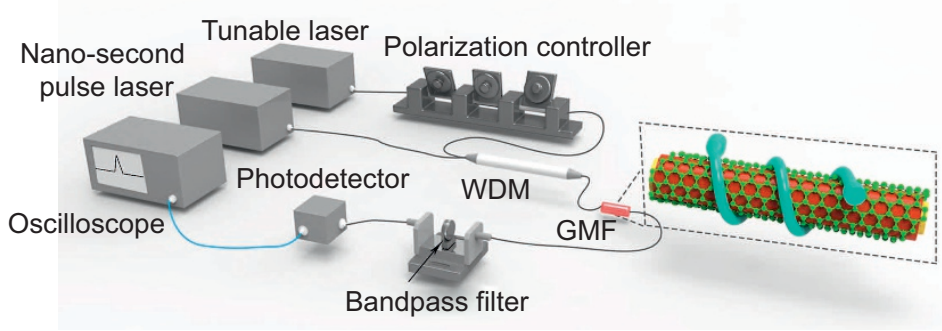

b

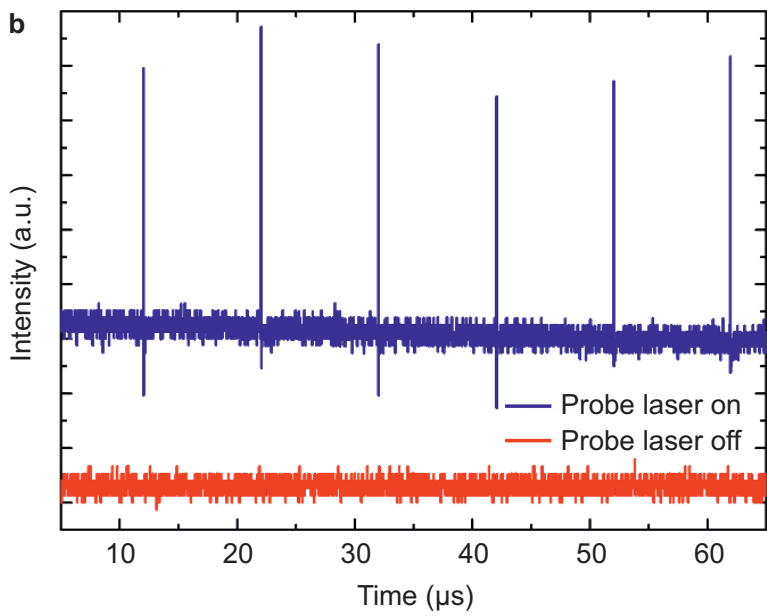

Figure 4 Pulse-laser pump probe system. (a) Optical measurement system. (b) Time-domain response of the GMF modulator. The modulated probe light curve was moved up for clarity.

GMF device. The modulation efficiency is the modulation depth of the GMF device per unit pump power. In Figure 3c, when the pump power reaches $35 \mathrm{~mW}$, the modulation depths of the HAM and LAM are 7.5 $\mathrm{dB}$ and $2.5 \mathrm{~dB}$, respectively, in the range of 1525-1565 $\mathrm{nm}$. Accordingly, the modulation efficiencies of the HAM and LAM are $\sim 0.2 \mathrm{~dB} \mathrm{~mW}^{-1}$ and $\sim 0.07 \mathrm{~dB} \mathrm{~mW}^{-1}$, respectively. The polarizationdependent characteristics can provide more options, and we can achieve different modulation depths (insertion losses) for different polarization states, depending on the specific requirements of the practical application. Moreover, we can use an in-line fiber polarizer to select the desired launched polarization of light and thereby avoid the extra noise caused by polarization mixing. In Figure 3d, we can see that the modulation depth of the GMF increases linearly with the pump power. To further illustrate the relation between the modulation depth and the pump power, we build a classical small-signal amplification model $^{20}$ to fit the experimental data (see Supplementary Information Note 1). It should be noted that the linear relation between the modulation depth and the pump power holds only for the small-signal condition. As the modulation depth increases with the pump power, it will reach a saturation value, and the linear relation will naturally break down. In our numerical calculation, the theoretical maximum modulation depth of our platform is $\sim 23 \mathrm{~dB}$ and $5.4 \mathrm{~dB}$ for the HAM and LAM, respectively (see Supplementary Information Note 2), for a 3.0$\mu \mathrm{m}$-diameter microfiber with a two-coil structure. For a $2.5-\mu \mathrm{m}$-diameter microfiber, the modulation depths of the HAM and LAM should be $42 \mathrm{~dB}$ and $9.6 \mathrm{~dB}$ ( $\mathrm{L} \sim 12 \mathrm{~mm})$, respectively. The modulation depth can be further improved if we optimize the coil number and microfiber diameter. Then the HAM can switch between the on-state and the offstate with an exceptionally large contrast, a fast speed and an unavoidable insertion loss from the intrinsic absorption.

As we can see, our current experimental results only reach one-third of the theoretical values at a $3.0 \mu \mathrm{m}$ diameter. One of the reasons is that the maximized pump power we used did not saturate over the entire GMF interaction length. The reason why we did not further increase the pump power in our experiment was that breakdown power threshold of the GMF existed because many residues of the protecting film of PMMA remained on the graphene surface after the PMMA removal process, which caused a great heating effect when launched with a high pump power. The average insertion losses for HAM and LAM are $\sim 10 \mathrm{~dB} /$ turn and $\sim 5 \mathrm{~dB} /$ turn, respectively, in our experiments, which are larger than the simulation results (Supplementary Information Note 2). We attribute this to the contamination of the graphene during the transfer process. If the fabrication method is perfected (for example, the PMMA can be fully removed, and only pure graphene will be left), the modulation depth of our GMF can be further improved to approach the theoretical value, which is mainly determined by the insertion loss from the intrinsic absorption. The higher modulation depth upper-limit always entails a higher insertion loss that depends on the light-graphene interactions. In practical applications, we must balance the interaction length between the insertion loss and the modulation depth based on real requirements.

To observe the time-domain response of the GMF modulator, we used a nanosecond laser (KEOPSYS, PYFL-K04-RP-030-006-0501064-T0-ET1-PK5D-FA) at $1064 \mathrm{~nm}$ to conduct the pump probe experiment. The pump probe system is illustrated in Figure $4 \mathrm{a}$. We used a bandpass filter centered at $1550 \mathrm{~nm}$ with a full width at half maximum (FWHM) of $12 \mathrm{~nm}$ to filter out the pump pulse laser. The modulated probe light was then detected by a photo-detector (New Focus, 1544-B), and the transformed electric signal could be analyzed by an oscilloscope (Agilent Technologies, DSO-X 4024A). When the pulse laser (pulse width of $\sim 6 \mathrm{~ns}$, repetition rate of $100 \mathrm{kHz}$ ) of average power $1.5 \mathrm{~mW}$ was launched into the GMF device along with a CW 1550-nm probe light (Agilent, tunable laser $81980 \mathrm{~A}$ ) of power $\sim 5$ $\mathrm{mW}$, the pulse laser increased the probe light transmission of the GMF device in their presence and switched off the probe source when they were absent. This can be readily seen in Figure $4 \mathrm{~b}$. When the probe laser was switched off, there was no modulated serial signal, which reflects the excellent filtering of the pump light. When the probe laser was turned on, a modulated probe light was clearly observed, which unambiguously demonstrated that our GMF device could serve as an all-optical modulator. Although we only demonstrated $100-\mathrm{kHz}$ modulation repetition, the GMF structure can be operated at sub$\mathrm{THz}$ repetition rates for the ultrafast carrier relaxation of graphene $\mathrm{e}^{14}$.

There is concern that the longer light-graphene interaction length may lead the pump and probe light to separate because of group velocity dispersion, thus limiting the fast response. We think that this problem may be inevitable if a high modulation depth is demanded because there is a balance between the response time and the modulation depth. Of course, there are several methods to ease the contradiction. First, it is convenient to tune the group velocity dispersion of a microfiber by coating a thin film, such as Teflon or polydimethylsiloxane (PDMS), onto the surface of the microfiber ${ }^{21}$. Second, we can choose a thinner 
microfiber and a thinner supporting rod to reduce the interaction length while achieving a reasonably good modulation depth.

\section{CONCLUSIONS}

Using a GMF-integrated stereo device, we demonstrated a highly polarization-sensitive all-optical modulating platform. The unique geometry practically enabled a sufficient light-graphene interaction length and strength, and a modulation depth of $\sim 7.5 \mathrm{~dB}(\sim 2.5 \mathrm{~dB})$ and modulation efficiency of $\sim 0.2 \mathrm{~dB} \mathrm{~mW}^{-1}\left(\sim 0.07 \mathrm{~dB} \mathrm{~mW}^{-1}\right)$ were simultaneously achieved for the HAM (LAM). The polarizationdependent modulation characteristics and time-domain response were also investigated. The performance was limited by the relatively low quality of the graphene used in the platform. A modulation depth as high as the theoretical prediction $(\sim 23 \mathrm{~dB}$ at a $3 \mu \mathrm{m}$ diameter, HAM) can be expected by improving the transfer and wrapping processes of the graphene. The findings of this study may contribute to many future applications in all-optical fiber-optic circuits.

\section{ACKNOWLEDGEMENTS}

This work is sponsored by the National 973 program (2012CB921803 and 2011CBA00205), the National Science Fund of China under grants 61225026, 61322503, 61457069, 61435008 and 61490714, and the Fundamental Research Funds for the Central Universities. The authors thank Hao Qian and Song-hua Cai for their assistance in drawing the schemes. The authors also thank the anonymous reviewers for their gracious comments, which helped us improve the paper.

1 Novoselov KS, Geim AK, Morozov SV, Jiang D, Zhang Y et al. Electric field effect in atomically thin carbon films. Science 2004; 306: 666-669.

2 Nair RR, Blake P, Grigorenko AN, Novoselov KS, Booth TJ et al. Fine structure constant defines visual transparency of graphene. Science 2008; 320: 1308 .

3 Li ZQ, Henriksen EA, Jiang Z, Hao Z, Martin MC et al. Dirac charge dynamics in graphene by infrared spectroscopy. Nat Phys 2008; 4: 532-535.
4 Gan XT, Shiue RJ, Gao YD, Mak KF, Yao XW et al. High-contrast electrooptic modulation of a photonic crystal nanocavity by electrical gating of graphene. Nano Lett 2013; 13: 691-696.

5 Schedin F, Geim AK, Morozov SV, Hill EW, Blake P et al. Detection of individual gas molecules adsorbed on graphene. Nat Mater 2007; 6: 652-655.

6 Wehling TO, Novoselov KS, Morozov SV, Vdovin EE, Katsnelson MI et al. Molecular doping of graphene. Nano Lett 2008; 8: 173-177.

7 Fang ZY, Wang YM, Liu Z, Schlather A, Ajayan PM et al. Plasmon-induced doping of graphene. ACS Nano 2012; 6: 10222-10228.

8 Bao QL, Zhang H, Wang Y, Ni ZH, Yan YL et al. Atomic-layer graphene as a saturable absorber for ultrafast pulsed lasers. Adv Funct Mater 2009; 19: 3077-3083.

9 Gan XT, Shiue RJ, Gao YD, Meric I, Heinz TF et al. Chip-integrated ultrafast graphene photodetector with high responsivity. Nat Photonics 2013; 7: 883-887.

10 Mueller T, Xia FN, Avouris P. Graphene photodetectors for high-speed optical communications. Nat Photonics 2010; 4: 297-301.

11 Xia FN, Mueller T, Lin YM, Valdes-Garcia A, Avouris P. Ultrafast graphene photodetector. Nat Nanotechnol 2009; 4: 839-843.

12 Liu M, Yin XB, Ulin-Avila E, Geng BS, Zentgraf T et al. A graphene-based broadband optical modulator. Nature 2011; 474: 64-67.

13 Gao YD, Shiue RJ, Gan XT, Li LZ, Peng C et al. High-speed electro-optic modulator integrated with graphene-boron nitride heterostructure and photonic crystal nanocavity. Nano Lett 2015; 15: 2001-2005.

14 Li W, Chen BG, Meng C, Fang W, Xiao Y et al. Ultrafast all-optical graphene modulator. Nano Lett 2014; 14: 955-959.

15 Liu ZB, Feng M, Jiang WS, Xin W, Wang P et al. Broadband all-optical modulation using a graphene-covered-microfiber. Laser Phys Lett 2013; 10: 065901.

16 Kou JL, Chen Y, Xu F, Lu YQ. Miniaturized broadband highly birefringent device with stereo rod-microfiber-air structure. Opt Express 2012; 20: 28431-28436.

17 Kou JL, Chen JH, Chen Y, Xu F, Lu YQ. Platform for enhanced light-graphene interaction length and miniaturizing fiber stereo devices. Optica 2014; 1: 307-310.

18 Ferrari AC, Meyer JC, Scardaci V, Casiraghi C, Lazzeri M et al. Raman spectrum of graphene and graphene layers. Phys Rev Lett 2006; 97: 187401.

19 Dawlaty JM, Shivaraman S, Chandrashekhar M, Rana F, Spencer MG. Measurement of ultrafast carrier dynamics in epitaxial graphene. Appl Phys Lett 2008; 92: 042116.

20 Yariv A, Yeh P. Optical Electronics in Modern Communications. 6th ed. Oxford: Oxford University Press; 2007: 230-232.

$21 \mathrm{Xu} \mathrm{ZY,} \mathrm{Li} \mathrm{YH,} \mathrm{Wang} \mathrm{LJ.} \mathrm{In} \mathrm{situ} \mathrm{fine} \mathrm{tailoring} \mathrm{of} \mathrm{group} \mathrm{velocity} \mathrm{dispersion} \mathrm{in} \mathrm{optical}$ microfibers via nanocoatings. Opt Express 2014; 22: 28338-28345.

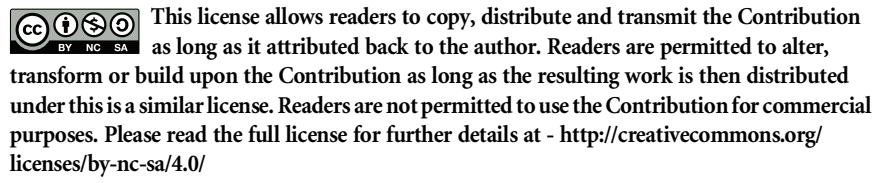

